PROSTATE CANCER

\title{
Equal access $=$ equal outcomes in black and white men
}

The observed phenomenon that black men have poorer outcomes than white men with prostate cancer has been investigated in a large multiple-cohort study, which suggests that the specific association between black race and prostate-cancerspecific mortality (PCSM) ceases to exist after adjustment for nonbiological variables.

"In the USA, black men have higher rates of PCSM than white men," corresponding author Daniel Spratt tells Nature Reviews Urology. "This difference is largely, if not entirely, secondary to social disparities that exist, and when you adjust for equal access to care and/or receiving standardized treatment, black and white men have similar long-term survival from prostate cancer."

As well as having poor prostate cancer outcomes, black men are also more likely to have comorbidities such as cardiovascular disease and diabetes, and a lower socioeconomic status. Thus, understanding whether their prostate cancer outcomes are an effect of different biology or socioeconomics is complicated.

Spratt's group addressed this difficulty by investigating PCSM, other-cause mortality (OCM) and all-cause mortality (ACM) differences between black and white men using three cohorts of patients diagnosed with nonmetastatic prostate cancer. Overall, 306,100 men were included in the study: 296,273 from the

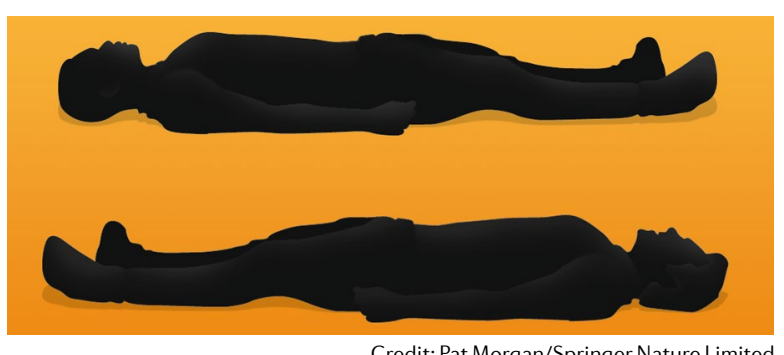

Credit: Pat Morgan/Springer Nature Limited
Surveillance, Epidemiology and End Results (SEER) database, 3,972 from 5 equal-access Veterans Affairs (VA) centres, and 5,854 from a pooled cohort from Radiation Therapy Oncology Group (RTOG) phase III randomized controlled trials. Data from these cohorts were collected and analysed using Fine-Gray subdistribution hazard ratio (sHR) models; inverse probability weighting (IPW) was used to adjust for imbalances by race and enable comparison.

Both the SEER and RTOG cohorts included similar proportions of black men $-17.8 \%$ and $19.3 \%$, respectively - whereas $38.1 \%$ of the VA cohort was black. Median age of black men was 2-3 years younger than white men across all cohorts. Percentages of high-risk disease were similar in the SEER and VA cohorts (22.1\% and $18.8 \%$, respectively), compared with $43.9 \%$ in the RTOG trials cohort. However, percentages of high-risk disease were higher in black men than white men across all three cohorts (1.8\% for SEER, $0.5 \%$ for VA and $5.2 \%$ for RTOG). Importantly, black men in the SEER cohort had a lower socioeconomic status (lowest quintile, $29.5 \%$ versus $17.7 \%$ of white men) and lower rates of non-Medicaid insurance $(61.9 \%$ of black men versus $64.6 \%$ of white men) than white men, and fewer black men received curative local treatment (76.3\% versus $79.1 \%$ ).

However, after IPW, age and stage adjustment, black men in the SEER cohort demonstrated an absolute increased PCSM of only $0.5 \%$ compared with white men at 10 years after diagnosis. Subgroup analysis revealed that the increased PCSM hazard in black men was only observed in men with low-risk and intermediate-risk disease, not in those with high-risk disease. By contrast, no significant difference in PCSM sHR by race was observed in the age-adjusted IPW models in the VA or RTOG cohorts. No significant difference in PCSM by race was found in the fully adjusted VA cohort and, in fact, in the fully adjusted RTOG cohort, black men had a lower PCSM sHR than white men and an absolute $2.2 \%$ lower rate of PCSM at 10 years. At the same time point, black men had an absolute increased OCM of $3.8 \%$ and $2.9 \%$ in the SEER and RTOG cohorts, respectively, driving a $4.4 \%$ increased $\mathrm{ACM}$ at the same time point.

As the most comprehensive analysis of race and prostate cancer performed thus far, this study indicates that socioeconomic factors, not differential biology, are the main drivers for the discrepant outcomes observed in black and white men with prostate cancer. Poorer overall health in black men might also be an important factor. "Black men with prostate cancer might have similar prostate cancer outcomes to white men, but they are substantially more likely to die from other diseases, like heart disease," explains Spratt. "This underscores that this greatest disparity for black men with prostate cancer has nothing to do with their cancer, but rather their overall health status. The biggest method to reduce disparities in overall survival between black and white men is to ensure equal access to quality care."

These data also raise some interesting questions about why this counterintuitive pattern arises. "Further work is needed to better understand why prostate cancer incidence is more common in black men, as if they have less access to care and undergo less frequent PSA screening, you would expect the opposite finding."

Annette Fenner

ORIGINAL ARTICLE Dess, R. T. et al. Association of black race with prostate cancer-specific and other-cause mortality. JAMA Oncol. https:// doi.org/10.1001/jamaoncol.2019.0826 (2019) 